\title{
ON A DISTRIBUTED CLUSTERING PROCESS OF COFFMAN, COURTOIS, GILBERT AND PIRET
}

\author{
J. VAN DEN BERG *** AND
}

A. ERMAKOV ${ }^{*}$ CWI

\begin{abstract}
Coffman et al. (1991) have introduced a flow process in graphs, where each vertex gets an initial random resource, and where at each time vertices with large resources tend to attract resources from neighbours. The initial resources are assumed to be i.i.d., with a continuous distribution.

We are mainly interested in the following question: does, with probability 1 , the resource of each vertex change only finitely many times?

Coffman et al. concentrate mainly on the case where the graph corresponds with the integer points on the line, in which case it is easily seen that the answer to the above question is positive. For higher-dimensional lattices they make general remarks which suggest that the answer to the above question is still positive. However, no proof seems to be known.

We restrict to the case of the square lattice, and, by a percolation approach, we reduce the question above to the question whether a certain quantity, which can be obtained from a finite computation, is sufficiently small. This computation is, however, still too long to be executed in an acceptable time. We therefore apply Monte Carlo simulation for this finite problem, which gives overwhelming evidence that, for the square lattice, the answer to the main question is positive.
\end{abstract}

Keywords: Flow of resources; clustering; percolation

AMS 1991 Subject Classification: Primary 60K35

Secondary 90B 15

\section{Introduction}

Coffman et al. [2] introduced the following model of 'distributed clustering'. Let $G$ be a (finite or countable), locally finite, connected graph.

We denote by $\rho(v, w)$ the graphical distance between the vertices $v$ and $w$ (i.e. the minimal number of edges in a path from $v$ to $w)$. Further, we define $B_{n}(v):=\{w: \rho(v, w) \leq n\}$, and $\partial B_{n}(v):=\{w: \rho(v, w)=n\}$.

Now assign to each vertex $v$ an initial 'resource' $r_{0}(v)$. We assume that these initial resources are non-negative i.i.d. random variables with a continuous distribution. Now we define the resources at time $t$, denoted by $r_{t}(v), t=1,2, \ldots$, inductively as follows. Let, for each $v$ with $r_{t}(v) \neq 0, a_{t}(v)$ be the vertex $w \in B_{1}(v)$ for which $r_{t}(w)$ is maximal (note that $a_{t}(v)$ may be $v$ itself). Now define, for each $v, r_{t+1}(v)=\sum_{w: a_{t}(w)=v} r_{t}(w)$. In other words,

Received 7 June 1996; revision received 27 October 1997.

* Postal address: CWI, Kruislaan 413, 1098 SJ Amsterdam, The Netherlands.

**Email address: j.van.den.berg@cwi.nl

Supported by the Netherlands Organization for Scientific Research (NWO). 
at each time each vertex (simultaneously with all others) gives its resource to the richest vertex in its neighbourhood. We are mainly interested in the following questions.

\section{Question 1.}

(a) Does, with probability 1 , each vertex eventually reach a final resource value?

(b) If the answer to question (a) is positive, is the expectation of this final value equal to the expectation of the initial value? (In other words: 'Is it impossible for resources to get lost'?).

\section{Remarks.}

1. Clearly, once a vertex has resource value 0 , its value remains 0 for ever. Further, it is not difficult to see that, if $G$ is finite, then at each time that at least one vertex changes its value, the value of some vertex changes from positive to 0 . Hence, on a graph with $n$ vertices, all resource values remain unchanged after time $n$.

2. Coffman et al. concentrate mainly on the case where $G$ is the graph of which the vertices are the integer points on the line, and where each pair of consecutive integers has an edge. For that case the evolution of the system is, in some sense, very regular. In particular, the answer to Question 1(a) and (b) is positive, and several quantities of interest can be explicitly computed.

3. As Coffman et al. pointed out (in the last section of their paper), the evolution in the higher-dimensional cases is typically much more complicated, and there is no hope for exact calculations as done for the line. Without explicitly saying so, they suggest that the answers to Question 1(a) and (b) above are still positive for these cases. However, it seems that no proof is known.

4. Van den Berg and Meester [5] prove weaker stability properties than those in Question 1 (a) and (b). In particular, for the square lattice they prove that (a.s.) for each vertex $v$, eventually either $r_{t}(v)=0$, or $a_{t}(v)$ remains constant. However, Question 1 remained open.

In the present paper we concentrate, like Van den Berg and Meester (see Remark 4 above) on the square lattice. However, we use a percolation-like approach (Van den Berg and Meester used ergodicity and symmetry arguments). In Section 2, by an adaptation of quite standard percolation arguments, we reduce Question 1 to a finite problem. Although this problem is finite, it appears to be too large to be solved rigorously in a realistic time. We therefore applied Monte Carlo simulation to it, which strongly convinced us that, for the square lattice, the answer to Question 1(a) and (b) is positive. As a side result, for the evolution on an $n \times n$ torus instead of the full lattice, the expected time until all vertices have reached their final value is at most of order $\log n$.

\section{Results}

In the remainder of this paper $G$ is the square lattice, i.e. the graph of which the vertices are the elements of $\mathbb{Z}^{2}$, and where two vertices $v=\left(v_{1}, v_{2}\right)$ and $w=\left(w_{1}, w_{2}\right)$ share an edge iff $\left|v_{1}-w_{1}\right|+\left|v_{2}-w_{2}\right|=1$.

Let $r_{0}(v), v \in \mathbb{Z}^{2}$, and $r_{1}(v), v \in \mathbb{Z}^{2}$ be the initial resources and the resources at time 1 respectively, as described in Section 1. In particular, the $r_{0}(v)$ are non-negative i.i.d. random variables with a continuous distribution. Further, let the random variables $X_{v}$ be defined by $X_{v}=1$ if $r_{1}(v)>0$, and 0 otherwise, $v \in \mathbb{Z}^{2}$. Note that the $X$-values depend only on the order statistics of the $r_{0}$-values. Also note that the $X$-process is 4-dependent: if $V, W \subset \mathbb{Z}^{2}$ 
and $\min \{\|v-w\|: v \in V, w \in W\}>4$, then the collection $\left(X_{i}, i \in V\right)$ is independent of the collection $\left(X_{j}, j \in W\right)$.

We are particularly interested in the percolative properties of this $X$-process. (For a quite general treatment of percolation, see Grimmett [3]). When a vertex $v$ has $X(v)=1$, we say that $v$ is open. An open path is a path in the graph of which every vertex is open. The set $\left\{v: X_{v}=1\right\}$ can be partitioned into maximal connected components. We say that the $X$-process percolates if one or more of these components are infinite. Note that the future evolution of the resources on a finite component $C$ no longer depends on the values outside $C$ (because all vertices on the outer boundary of $C$ have resource 0 at time 1 and hence, as mentioned in Section 1, Remark 1, will have resource 0 for ever; this boundary with 0 resource values isolates $C$ from the outside). Hence (see again Section 1, Remark 1) after time $|C|+1$ the resource values of $C$ remain unchanged. So if, with probability $1, X$ does not percolate, then the answer to Question 1(a) is positive. Moreover, we then also have that no resource 'escapes to infinity' and hence (by standard arguments) that the answer to Question 1(b) is also positive.

To state the theorem below, we need some extra terminology and notation. Let $k \neq l$ be positive integers. By a crossing of the rectangle $([0, k] \times[0, l]) \cap \mathbb{Z}^{2}$ we mean an open path which starts on one of the long sides of the rectangle and ends on the opposite long side, and lies entirely in the rectangle.

By $p_{n, m}$ we denote the probability that there exists a crossing of a given $n \times m$ rectangle, say the rectangle $([0, n] \times[0, m]) \cap \mathbb{Z}^{2}$.

We say that a function $f: \mathbb{N} \rightarrow \mathbb{R}^{+}$decays exponentially if $\exists \lambda>0$ such that $f(n)<\mathrm{e}^{-\lambda n}$, $n \in \mathbb{N}$.

Theorem 1. If there exist $N>0, K>4$ with $p_{N, 2 N+K}<1 / 13$, then (a)-(d) below hold.

(a) The probability of an open path from $O$ to $\partial B_{n}$ decays exponentially in $n$. In particular, the X-process does not percolate and the answer to Question 1( $a$ ) and ( $b$ ) (in Section 1) is positive.

(b) $P(|C|>n)$ decays exponentially in $n$, where $C$ is the open component of the origin.

(c) $P(\tau>n)$ decays exponentially in $n$, where $\tau$ is the smallest time after which the resource value of $O$ remains unchanged.

(d) Consider the distributed clustering process on an $n \times n$ torus (instead of the full square lattice), and let $T$ be the smallest time after which all resource values remain unchanged. Then $E(T)=O(\log n)$.

\section{Remarks.}

1. It is a well-known result in the percolation literature that if crossing probabilities are smaller than a certain $\kappa$, then no percolation occurs and the cluster radius and cluster size distributions have an exponential decay. However, in that literature one is mainly interested in the existence of such $\kappa$ rather than its value. This explains why the values which arise from the computations in the early publications were extremely small (see the proof of Theorem 1(b)). Later, in the work of Chayes and Chayes [1] a scaling argument was used to show that $1 / 16$ suffices. Below, we refine the technique used in [4] and obtain the threshold of $1 / 13$. In the dimensions higher than 2 , our method produces thresholds on the crossing probabilities, which are larger by an order of magnitude than those obtained by the argument of Chayes and Chayes. 


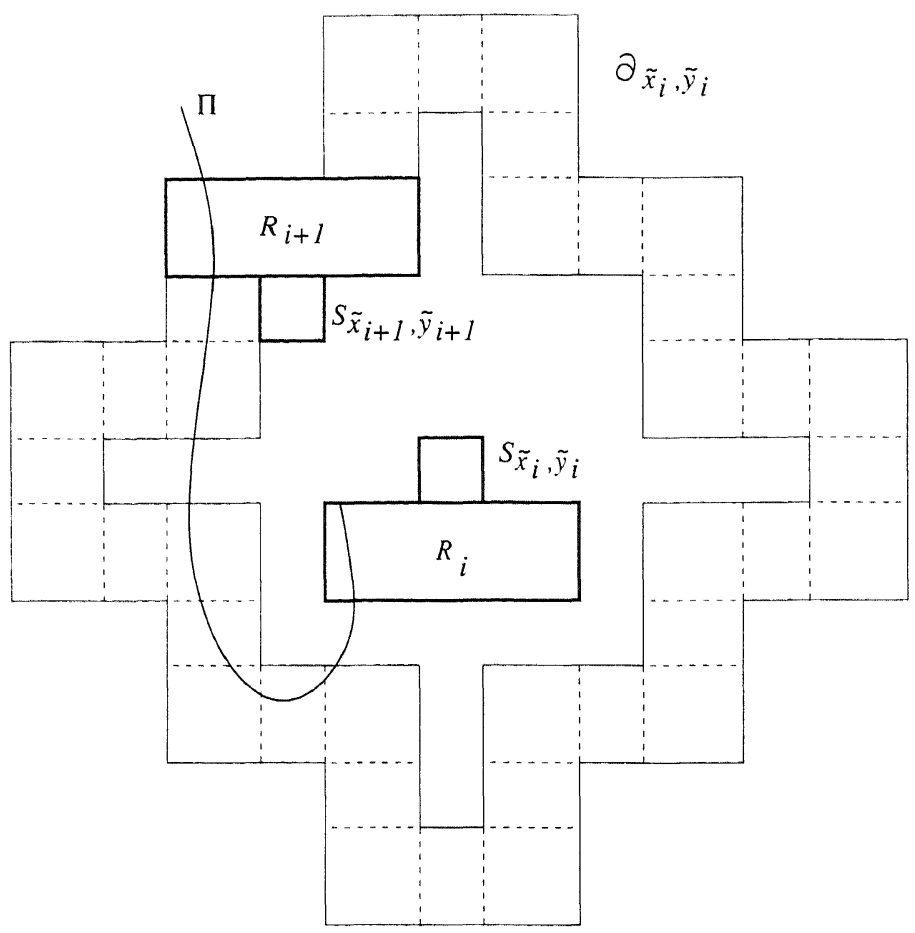

FIGURE 1: A part of a path $\Pi$, which crosses the rectangles $R_{i}$ and $R_{i+1}$. The second rectangle is an element of $\partial_{\bar{x}_{i}, \bar{y}_{i}}$.

2. Since $p_{n, 2 n+k}$ is non-decreasing in $k$, it makes sense to check the conditions of Theorem 1 only for $K=5$, the smallest value beyond the radius of dependence of the $X$ process.

Proof of Theorem 1. First we need some more notation. Let $N$ and $K$ be fixed and satisfy the condition of the theorem. Let $\alpha(i)=\lfloor i / 2\rfloor(K+N)+\mathbf{1}_{\{i \text { is odd }\}} K, i \in \mathbb{Z}$. Let, for integer $n, m, S_{n, m}$ be the rectangle $([\alpha(n), \alpha(n+1)] \times[\alpha(m), \alpha(m+1)]) \cap \mathbb{Z}^{2}$. In the simplest case of $K=N, \alpha(i)=N i$, and all $S_{n, m}$ are $N \times N$ squares. In the general case, if both indices $n$ and $m$ are even, or both are odd, or one is even and the other is odd, $S_{n, m}$ is a $K \times K, N \times N$ or $N \times K$ rectangle respectively. The union of all $S_{n, m}$ covers the integer lattice, and each two of them are either disjoint or share a common side.

Let $H_{n, m}$ be the horizontal rectangle given by $H_{n, m}=S_{n-1, m} \cup S_{n, m} \cup S_{n+1, m}$. We call $n, m$ the coordinates of $H_{n, m}$. Further let $V_{n, m}$ be the vertical analog of $H_{n, m}$, i.e. $V_{n, m}=$ $S_{n, m-1} \cup S_{n, m} \cup S_{n, m+1}$. Finally, for $n$ and $m$ even, let $\partial_{n, m}$ be the set of twenty $(2 N+K) \times N$ rectangles which 'surrounds' $S_{n, m}$ at 'scaled distance' 5 (see Figure 1). More precisely,

$$
\partial_{n, m}=\left\{H_{n+i, m+j}, V_{n+j, m+i}:|i|+|j|=5, i \text { is even }\right\} .
$$

Proof of Theorem I( $a$ ). Suppose there is an open path $\Pi$ from $O$ to $\partial B_{n}$. If $n$ is sufficiently large, compared to $N$ and $K$, $\Pi$ goes outside $\cup_{-6 \leq i, j \leq 6} S_{i, j}$. Hence there must be a crossing of some rectangle belonging to $\partial_{0,0}$. Let $R_{1}$ be the rectangle which corresponds to the last of such crossings appearing on the path $\Pi$ (it is not difficult to check that this is well defined), 
and let $\left(x_{1}, y_{1}\right)$ be the coordinates of $R_{1}$. Remark: it is important to note that after this last crossing $\Pi$ will never visit the set $\bigcup_{|i|,|j| \leq 3} S_{i, j}$ again. Let $\tilde{x}_{1}$ and $\tilde{y}_{1}$ be two even numbers such that $\left|\tilde{x}_{1}\right|+\left|\tilde{y}_{1}\right|=4$ and $\left|x_{1}-\tilde{x}_{1}\right|+\left|y_{1}-\tilde{y}_{1}\right|=1$. So $\left(\tilde{x}_{1}, \tilde{y}_{1}\right)$ are the coordinates of the $K \times K$ square $S_{\tilde{x}_{1}, \tilde{y}_{1}}$ neighbouring $R_{1}$ at the side of the origin. Now, as above, if $n$ is sufficiently large there must be a crossing of a rectangle in $\partial_{\tilde{x}_{1}, \tilde{y}_{1}}$. Let $x_{2}, y_{2}$ be the coordinates of the rectangle $R_{2}$ corresponding to the last such crossing. By the remark above, each vertex of this crossing is at distance $\geq K$ from $R_{1}$. Carrying on like this we construct a sequence of rectangles $R_{1}, R_{2}, \ldots, R_{k}$ with $k=\lfloor n /(2 N+2 K)\rfloor-1$, and a sequence $\left(\tilde{x}_{i}, \tilde{y}_{i}\right), i=0,1, \ldots, k$ such that

(i) $\tilde{x}_{0}=\tilde{y}_{0}=0 ; R_{i} \in \partial_{\tilde{x}_{i-1}, \tilde{y}_{i-1}}, 1 \leq i \leq k ; \tilde{x}_{i}$ and $\tilde{y}_{i}$ are even and such that $\left|\tilde{x}_{i}-\tilde{x}_{i-1}\right|+$ $\left|\tilde{y}_{i}-\tilde{y}_{i-1}\right|=4$ and $\left|x_{i}-\tilde{x}_{i}\right|+\left|y_{i}-\tilde{y}_{i}\right|=1$, where $\left(x_{i}, y_{i}\right)$ are the coordinates of $R_{i}, 1 \leq i \leq k$.

(ii) There is a crossing of $R_{1}$; for each $i, 2 \leq i \leq k$, there is a crossing of $R_{i}$ which has distance $\geq K$ from each $R_{j}, j<i$.

Note that, since $K$ is larger than 4 (and the $X$-process is 4-dependent), the events in (ii) are independent. Each of these events has probability $\leq p_{N, 2 N+K}$. Further, $\partial_{\bar{x}_{i}, \tilde{y}_{i}}$ has 20 elements (rectangles) for any $i$, but if $i \geq 1$ then, as the reader can check, at least 7 of them are covered or surrounded by $\partial_{\tilde{x}_{i-1}, \tilde{y}_{i-1}}$. So there are at most 13 'fresh' rectangles, and at least one of them is crossed by the path $\Pi$. Hence, there are at most $20 \times 13^{k-1}$ sequences which satisfy (i). Hence, the probability that there exists an open path from $O$ to $\partial B_{n}$ is at most $20 \times 13^{k-1} p(N)^{k}$, with $k$ as defined above. From this the result follows immediately.

Proof of Theorem $I(b)$. As mentioned before, in the literature on percolation there are theorems analogous to 1 (b) but with the condition ' $\exists N: p_{N, 2 N+K}<1 / 13$ ' replaced by ' $\exists N: p_{N, 3 N}<\kappa$ ', where $\kappa$ is a fixed small number (for instance, the $\kappa$ in Theorem 1 of Kesten [4] is (in the case of the square lattice) smaller than $10^{-100}$ ). The proof of these theorems can be straightforwardly adapted (possibly at the cost of an even smaller $\kappa$ ) to finite-dependent models. However, the smallness of $\kappa$ is no longer important to us, because $p_{m, 3 m}$ is clearly at most the product of the number of vertices on a long side of a given $3 m \times m$ rectangle (i.e. $3 m+1$ ) and the probability of an open path from $O$ to $\partial B_{m}$, which by 1(a) goes to 0 as $m$ goes to $\infty$.

Proof of Theorem $I(c)$. This follows immediately from $l(b)$ and the earlier observation that $\tau \leq|C|+1$.

Proof of Theorem $l(d)$. Note that, for $l$ sufficiently small (compared to $n$ ), the distribution of the configuration at time 1 on a set of vertices of radius $l$ 'does not feel' whether we work on an $n \times n$ torus or on the full lattice. In particular, the probability that the component of $O$ is smaller than $l$ is then the same for the torus as for the infinite lattice. Let, for each vertex $v$ on the torus, $T_{v}$ be the smallest time after which its resource value remains unchanged. By the observation in Remark 1 in Section $1, T \leq$ the size of the $n \times n$ torus, which is $n^{2}$. Therefore we trivially have, for each $a>0$,

$$
E T=\sum_{l=1}^{n^{2}} P(T \geq l) \leq a \log n+n^{2} P(T \geq a \log n) .
$$


Further, $n^{2} P(T \geq a \log n)=n^{2} P\left(\exists\right.$ a vertex $v$ on the torus with $\left.T_{v} \geq a \log n\right) \leq n^{4} P\left(T_{O}\right.$ $\geq a \log n$ ). However, by the observation at the beginning of this proof, for each $a$, for all sufficiently large $n$ (depending on $a), P\left(T_{O} \geq a \log n\right)=P(\tau \geq a \log n)$, with $\tau$ as in 1 (c). From this and 1(c), we obtain that, for $a$ sufficiently large, the second term on the right-hand side of (1) goes to 0 as $n \rightarrow \infty$.

This completes the proof of Theorem 1 .

Conjecture. $p_{5,15}<1 / 13$.

Remark. Clearly (by Theorem 1), under this conjecture the conclusions in Theorem 1(a)-(d) hold (and hence the answers to Question 1(a) and (b) are positive).

Although we have not computed a rigorous convenient upper bound for $p_{5,15}$, the word 'conjecture' is somewhat too weak here. One should note that the exact computation of $p_{5,15}$ is a finite task. As we remarked before, the $X$-process depends only on the order statistics of the initial resource process. More precisely, the $X_{i}, i \in H_{0,0}$, depends only on the order statistics of the $r_{0}(j), \rho\left(j, H_{0,0}\right) \leq 2$. So the exact computation of $p_{5,15}$ can be done by enumerating all permutations of the set $\{1,2, \ldots,|W|\}$, where $W=\left\{j: \rho\left(j, H_{0,0}\right) \leq 2\right\}$, and counting the number of permutations which give rise to a crossing of $H_{0,0}$ in the induced $X$-configuration. However, since $|W|>100$, not even the fastest computer is able to do this in a realistic time. Maybe by clever inclusion-exclusion arguments one could obtain a rigorous upper bound $<1 / 13$ for $p_{5,15}$ (or some other $p_{n, 2 n+5}$ ). However, we have not done this, but instead used Monte Carlo simulation to estimate $p_{n, 2 n+5}$ for various values of $n$ (see Table 1 below and compare the crossing probabilities with $1 / 13=0.07692 \ldots$ ), which very strongly indicates that the conjecture is true.

TABLE 1.

\begin{tabular}{cccc}
\hline & $\begin{array}{c}\text { Monte Carlo estimate } \\
\text { of } p_{n, 2 n+5}\end{array}$ & Standard deviation & \# simulations \\
\hline 4 & 0.13048 & 0.00005 & $5 \times 10^{7}$ \\
5 & 0.06798 & 0.00004 & $5 \times 10^{7}$ \\
6 & 0.03467 & 0.00003 & $5 \times 10^{7}$ \\
\hline
\end{tabular}

Final remark. Even if there were faster computers which could compute (or sufficiently bound) $p_{5,15}$ rigorously, the proof would still not be entirely satisfactory, because for other lattices one would have to make all the calculations again. It would be nice to have a more elegant and general proof of absence of percolation for the $X$-process, for instance by showing (in some sense) domination by a subcritical 'ordinary' percolation process. However, in spite of serious attempts, we have not succeeded in doing this.

\section{References}

[1] Chayes, J. T. And Chayes, L. (1986). Percolation and random media. Critical Phenomena, Random systems, Gauge theories (Les Houches, Session XLIII, 1984). Elsevier, Amsterdam, pp. 1001-1142.

[2] Coffman, E. G., Courtois, P. J., Gilbert, E. N. And Piret, P. (1991). A distributed clustering process. J. Appl. Prob. 28, 737-750.

[3] GrimmetT, G. (1989). Percolation. Springer, Berlin.

[4] KESTEN, H. (1981). Analyticity properties and power law estimates of functions in percolation theory. J. Statist. Phys. 25, 717-756.

[5] VAN DEN BERG, J. AND MeEster, R. W. J. (1991). Stability properties of a flow process in graphs. Random Structures and Algorithms 2, 335-341. 